P. LAVIALle. - SUR L ÉPIDERME INTERNE DU TÉguMENT DES COMPOSÉES. 75.

tant sous forme, soit de tissu criblé, soit d'îlots de tissu cribrovasculaire, provenant du morcellement des extrémités de l'arc principal, ou encore de tissu criblé détaché de cet arc et entouré de tissu ligneux différencié dans la zone médullaire, soit des fragments libéro-ligneux accompagnés de paquets de fibres protectrices; elles proviennent toujours de l'arc principal.

Le fait de présenter l'un ou l'autre de ces amas n'est pas caractéristique d'un genre ou mème d'une espèce. Sur la même plante, on peut très bien rencontrer des amas de tissu criblé ou des amas de tissu cribro-vasculaire. Il faut pourtant remarquer que le Vitex Agnus-castus L., présente seulement des îlots de tissu criblé et que dans le Vitex Negundo L., presque toujours, il existe du parenchyme lignifié autour des amas de tissu criblé ou de tissu cribro-vasculaire qui se trouvent ainsi fortement protégés.

Dans cette note, nous avons volontairement laissé de còté le cas des Avicenniées dont les anomalies de structure ont été exposées par Van Tieghem ${ }^{1}$.

En résumé, il n'existe donc pas de tissu criblé périmédullaire chez les Verbénacées et l'expression liber interne employée pour désigner ces îlots dont nous avons donné l'origine est donc impropre, puisque ces amas ne proviennent jamais d'une différenciation propre, mais d'une fragmentation de l'are vasculaire normal.

\title{
Sur le rôle digestif de l'épiderme interne du tégument oviulaire des Composées
}

\author{
PAR M. PIERRE LAVIALLE.
}

Hegelmaier ${ }^{2}$ attribue un simple rôle de protection à la “ couche épithéliale », c'est-à-dire à l'assise interne différenciée du tégument ovulaire, qui tapisse le sac embryonnaire des Composées.

1. Van Treghem, Avicenniées et Symphorémacées (Journ. de Bot. Morot, 1898, p. 345-365).

2. Hegelmaier, Ueber der Keimsack einiger Compositen (Bot. Zeit., $\mathrm{n}^{\circ} 50$, p. 805,1889$)$. 
M. Guignard ${ }^{1}$ et $M^{\text {He }}$ Goldflus ${ }^{2}$ attribuent à cette même assise un rôle digestif. Moi-même je suis arrivé à une conclusion identique $^{3}$. Voici les faits que j'ai observés et qui paraissent légitimer suffisamment cette manière de voir :

1 L L'épiderme du tégument ovulaire des Composées présente des caractères identiques dans la région interne, au contact du nucelle, et dans la région externe (surface de l'ovule), jusqu'au moment de la différenciation de la cellule-mère définitive du sac embryonnaire.

$2^{\circ}$ Le tissu du tégument compris entre les épidermes externe et interne reste parfaitement homogène jusqu'au moment de la différenciation de l'épiderme tégumentaire au contact du sac.

$3^{\circ}$ Pendant le développement du sac, on observe des cloisonnements radiaux accompagnés d'un allongement radial des cellules de la région épidermique du tégument qui est, très précisément, au contact du nucelle en voie de résorption, ou au contact du sac en voie de développement. Les autres régions de ce même épiderme ne sont l'objet d'aucune différenciation.

$4^{\circ}$ Immédiatement après cette différenciation des cellules qui tapissent le sac, avant même que ce dernier ait atteint son volume définitif, et uniquement au contact précis de la région différenciée qui s'étend de la limite interne du canal micropylaire jusqu'aux antipodes, le tégument ovulaire se montre envahi progressivement par un agent de désagrégation, de dissolution, qui divise le tégument en deux zones : la zone externe qui garde sa structure, la zone interne qui se présente sous la forme d'une sorte de gelée au sein de laquelle on ne décèle bientôt plus ni noyaux ni membranes.

$5^{\circ} \mathbf{L a}$ digestion de la zone interne du tégument se fait progressivement dans un sens centrifuge.

Les faits qui précèdent imposent l'idée de l'intervention d'une diastase sécrétée par la partie différenciée de l'assise qui

1. Guignard (L.), Rechtrches sur le développement de la graine et en particulier du tégument séminal (Journ. de Bot. Morot, Paris, 1893, p. 306).

2. Goldflus (Mathilde), Sur la structure et les fonctions de l'assise épithéliale et des antipodes chez les Composées (Journ. de Bot. Morot, 1898-1899).

3. LAvialle (P.), Recherches sur le développement de l'ovaire en fruit chez les Composées (Thèse Doctorat ès sciences nat., Paris, 1912). 
P. LAVIALle. - SUR L'ÉPIDER IN INTERNE DU TÉgument des CONPOSÉES. 77

m’occupe, que j’ai désignée, pour simplifier, sous le nom d'assise digestive.

M'inspirant des recherches de M. Guignard sur la localisation des principes actifs et des ferments solubles dans un certain nombre de familles ${ }^{1}$, je me suis demandé si les cellules de cette assise ne joignaient pas à leur apparence spéciale des réactions particulières.

Matières colorantes. - Parmi les matières colorantes qui se fixent avec une énergie particulière sur le contenu des cellules de l'assise digestive, je citerai : le brun Bismarck, le vert d'iode, le vert de méthyle.

Réactif de Millon. - J'ai essayé de distinguer par le réactif de Millon (nitrate acide de mercure) les cellules de l'assise digestive de celles appartenant aux régions non différenciées de l'épiderme tégumentaire. Ceci, dans des conditions rappelant celles où opérait M: Guignard dans ses recherches sur la localisation de la myrosine chez les Crucifères, etc., ou de l'émulsine dans les Amandes et la feuille du Laurier-Cerise.

Mes matériaux d'étude étaient constitués par des ovaires de Ceniaurea cyanus pris dans des capitules cueillis avant la fécondation.

Des coupes transversales et longitudinales pratiquées au niveau du sac dans les ovaires frais, immergées dans le réactif de Millon, prennent à froid une légère coloration rose dans toute leur étendue. Si on prolonge l'action du réactif et surtout si on élève graduellement la température en portant la préparation sur une platine chauffante, on constate que le contenu des cellules de l'assise digestive qui bordent le sac prend une coloration noir rougeâtre. En continuant à chauffer jusqu'à émission de vapeurs visibles, on voit l'intensité générale de la coloration diminuer peu à peu et la teinte rouge dominer.

Les tissus environnants prennent et gardent, dans les conditions précédemment décrites, une teinte rose très peu accentuée.

1. Guignard (L.), Recherches sur la localisation des principes actifs des Crucifères (Journal de Bot. Morot, Paris, 1890); ID., Sur la localisation, dans les Amandes et le Laurier.Cerise, des principes qui fournissent l'acidecyanhydrique (Journ. de Pharm. et de Chimie, 1890.); ID., Recherches sur la nature et la localisution des principes actifs chez les Capparidèes, Tropéolées, Limnanthées, Résédacées et Papayacées (Journ. de Bot. Morot, Paris, 1894). 
La zone interne gélifiée du tégument ovulaire, bien que contenant, selon toute vraisemblance, le ou les ferments sécrétés par l'assise digestive, non seulement ne se colore pas en noir rougeâtre, mais prend une coloration rose plus faible encore que celle développée par le réactif dans la zone externe.

Il est important de remarquer ici :

a) que la coloration intense communiquée aux cellules de l'assise digestive par le réactif de Millon ne paraît pas due à l'action de ce réactif sur le ou les ferments (la zone interne du parenchyme tégumentaire ne se colorant pas sensiblement), mais à la condensation particulièrement grande, peut-être même à la nature de la matière albuminoïde qui constitue le protoplasme des cellules ${ }^{1}$. Les résultats obtenus dans les essais de plasmolyse décrits plus loin appuient cette interprétation.

b) que la coloration est réservée exclusivement aux cellules de l'assise digestive. Toutes les autres régions non différenciées de l'épiderme tégumentaire, le canal micropylaire compris, se colorent légèrement en rose comme le tissu du tégument situé entre l'épiderme externe et l'épiderme interne.

Plasmolyse. - Les coupes d'ovules soumises à la plasmolyse, à l'aide d'une solution de nitrate de potasse à $100 / 0$, permettent de constater que la contraction du protoplasme est beaucoup plus intense pour les cellules périphériques du parenchyme tégumentaire que pour l'assise digestive. Pour cette dernière, en effet, le protoplasma se décolle à peine de la membrane cellulaire : ceci paraît montrer que la teneur en matière albuminoïde est plus élevée, que la teneur en eau est plus faible et explique, en partie au moins, la différence d'intensité de coloration obtenue avec le réactif de Millon, entre l'assise digestive et les autres régions de l'ovule.

Conclusions. - Le phénomène de la plasmolyse montre que le contenu des cellules de l'épiderme du tégument ovulaire qui tapissent le sac embryonnaire est plus dense, plus pauvre en eau, que le contenu des autres cellules du tégument.

1. Cette matière albuminoïde est peut-être très riche en tyrosine : on sait que, seuls, les albuminoïdes fournissant de la tyrosine dans leur dédoublement donnent une coloration rouge sous l'influence du réactif
de Millon. 


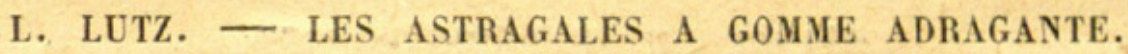

Le réactif de Millon donne une coloration rouge foncé, ou même rouge noirâtre au contenu des mêmes cellules. Cette coloration est absolument locale : le reste de l'épiderme et le parenchyme tégumentaire demeurent sensiblement incolores ou prennent une teinte rose très peu accentuée.

Ces faits rappellent les observations rapportées par M. Guignard dans ses travaux sur la localisation de la myrosine chez les Crucifères, etc., et de l'émulsine dans les Amandes et la feuille de Laurier-Cerise. Unis aux données fournies par l'étude histologique et par la succession des faits dans l'ordre chronologique :

a) différenciation de l'assise digestive,

b) gélification de la zone interne du tégument ovulaire,

c) direction centrifuge de la gélification,

ils donnent la conviction presque absolue que l'assise interne du tégument qui tapisse le sac sécrète des diastases qui se répandent dans la zone interne du tégument pour la digérer.

L'orifice interne du canal micropylaire constitue, à la fois, la limite supérieure de la différenciation de l'épiderme tégumentaire en assise digestive, et la limite de la résorption du tégument ovulaire dans cette région.

\title{
Nouvelles observations sur les Astragales à gomme adragante
}

\author{
PAR M. L. LUTZ.
}

Au cours de la Session de 1914, en Algérie, nous avons trouvé, notre collègue $\mathrm{R}$. Maire et $\mathrm{moi}^{1}$, un peuplement d'A canthyllis numidica Pomel producteur de gomme adragante.

Depuis lors, en mars 1924, notre collègue M. Bois a eu l'amabilité de récolter à mon intention des échantillons d'Astragalus armatus Willd. aux environs de Biskra. Une section transversale de la racine pivotante de ces échantillons montre

1. MAIRE (R.) et LutZ (L.), Sur la production de gomme adragante par $l$ 'Acanthyllis numidica Pomel (Bull. Soc. bot. Fr., LXI, 1914, sess. extr., p. $x \times x i v)$. 


\section{$2 \mathrm{BHL}$ Biodiversity Heritage Library}

Lavialle, Pierre. 1922. "Sur le rôle digestif de l'épiderme interne du tégument ovulaire des Composées." Bulletin de la Société botanique de France 69, 75-79. https://doi.org/10.1080/00378941.1922.10833407.

View This Item Online: https://www.biodiversitylibrary.org/item/93160

DOI: https://doi.org/10.1080/00378941.1922.10833407

Permalink: https://www.biodiversitylibrary.org/partpdf/161252

\section{Holding Institution}

Missouri Botanical Garden, Peter H. Raven Library

\section{Sponsored by}

Missouri Botanical Garden

\section{Copyright \& Reuse}

Copyright Status: Public domain. The BHL considers that this work is no longer under copyright protection.

This document was created from content at the Biodiversity Heritage Library, the world's largest open access digital library for biodiversity literature and archives. Visit BHL at https://www.biodiversitylibrary.org. 